\title{
Recurrence of spinal epidural abscess after inadequate antibiotic dosage regimen
}

\author{
Ram Krishna Dahal, MS; Javed Ahmad Khan, MS; Binod Bijukachhe, MS \\ Spine services, Department of Orthopedics, Grande International Hospital, Kathmandu, Nepal
}

Corresponding author

Ram K Dahal, MS

Email: dahalramk@gmail.com

Received 10 Dec 2018

Accepted 18 Dec 2018

ABSTRACT
Introduction
Spinal epidural abscess (SEA) is a potentially life threatening condition which can present with a
clinical triad of fever, back pain and neurological deficit. It is more common in elderly population with
risk factors like immunocompromised status, diabetes mellitus and alcoholism. High index of clinical
suspicion of SEA warrants prompt investigated and treatment. Immediate surgical decompression
along with antibiotic is recommended for better outcome.
Case Presentation
A 16 years old boy presented with complaints of fever, upper back pain which had developed gradually
over a period of 10 days. He had history of furuncle in perineal region 3 weeks prior to his symptoms,
which was drained. With high clinical suspicion of SEA, Contrast enhanced Magnetic Resonance Imaging
(CEMRI) was done along with blood parameters and was diagnosed as SEA D3-D6 level. He underwent
immediate surgical decompression and was given 3 weeks of antibiotics. Post-operative period was
uneventful with clinical improvement of the symptoms. He again presented to us with relapse of
the infection on $28^{\text {th }}$ post-operative day with similar fever and back pain. He was reinvestigated with
MRI and blood investigations which revealed relapse of the infection. He was then managed with
intravenous (IV) Vancomycin for 2 weeks and oral Erythromycin for another 6 weeks. He did not require
any surgical intervention in his later presentation.
Conclusion
Spinal epidural abscess is a surgical emergency and prompt diagnosis with surgical drainage is
recommended for better outcome even when there is no neurological deficit. Abscess drainage should
be adjunct with longer duration of antibiotics for at least 6 weeks for optimum results.
Keywords: Antibiotic, Immunocompromised, Spinal epidural abscess

\section{Introduction}

Spinal Epidural abscess (SEA) is a very serious cause of back pain which can result in rapid neurological deterioration and can result in a life threatening condition if it is not timely identified and treated. It is more common in $5^{\text {th }}-6^{\text {th }}$ decade of life ${ }^{1}$. Patients with preexisting immune-compromised status, intravenous drug abuse, alcoholism and diabetes mellitus are more susceptible to develop this condition ${ }^{1}$. Neuro-stimulation, epidural catheterizations or epidural injections are iatrogenic causes of SEA. Back pain and fever are common symptoms which may lead to rapid neurological deterioration - bowel/bladder incontinence, and sepsis. Treatment includes surgical decompression and drainage in combination with antibiotics ${ }^{2}$.

\section{Case Report}

The patient was a 16 years old otherwise healthy boy who presented to our emergency department with the complaints of occasional fever, and upper back pain which had developed over a period of 10 days. The intensity of pain was gradually increasing and was severe at the time presentation. X-rays of the dorsal spine was apparently normal. He was managed with analgesics and antipyretics in another center for fever and myalgia. 


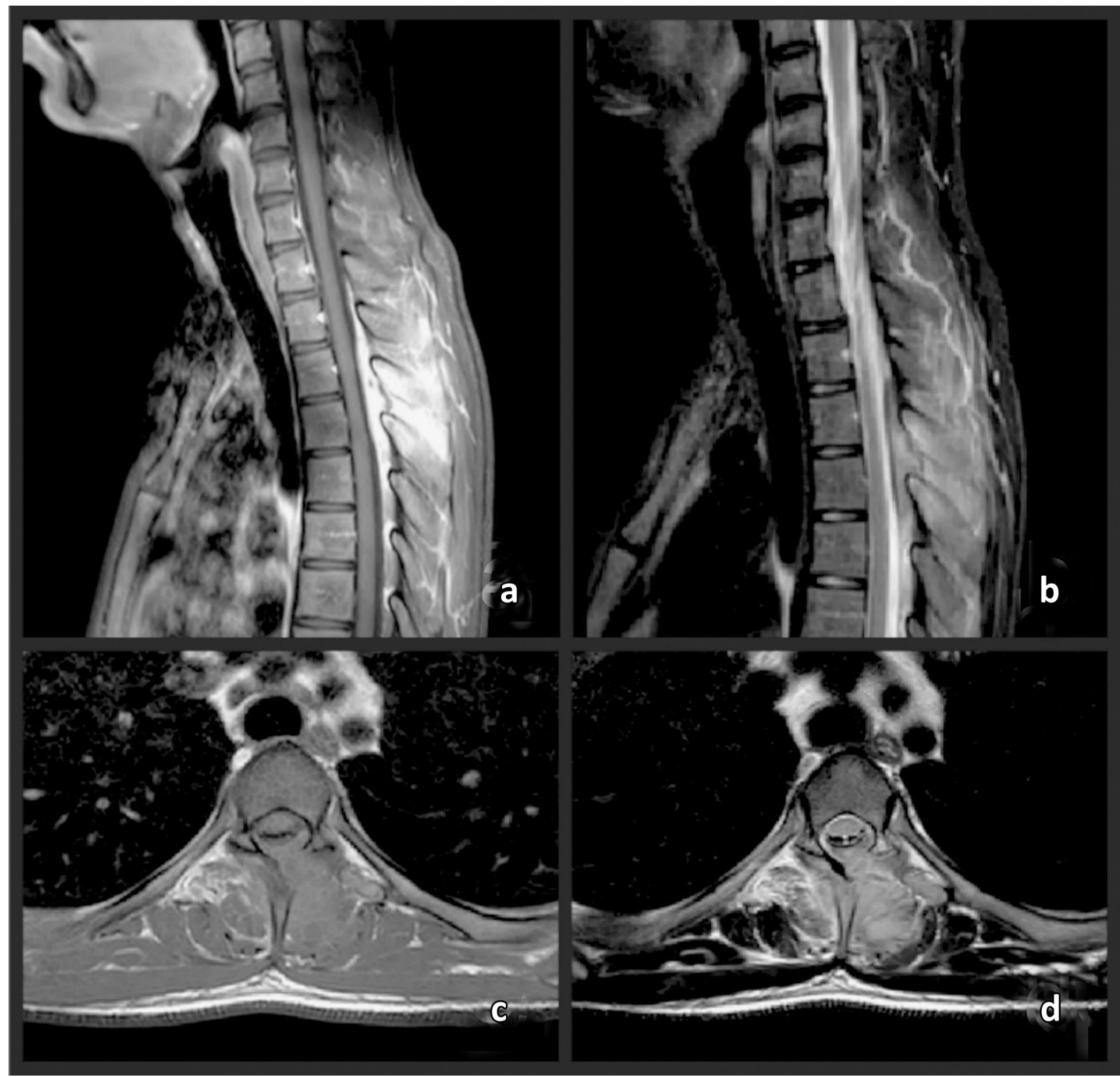

Figure 1: a,c - T1W sagittal/axial image of dorsal spine, Marrow signal changes in D3 and D4 spinous processes with heterogeneously enhancing signal changes in interspinous and paraspinal region along with peripherally enhancing paraspinal collection at D3 to D6 levels predominantly on the left side and enhancing soft tissue in posterior epidural space at D1 to D8 level causing mild thecal sac compression at D3/D4 level.

b,d - T2W sagittal/axial image at the same level as Fig. 1.a,c. MRI features suggestive of infective pathology with paraspinal abscess and inflammatory/infective tissue in posterior epidural space.

On clinical examination, he was not able to bear weight or lie supine. His past medical history was not significant and he had no history of previous/ recent trauma, spine surgery or immunodeficiency status. However, he gave history of furuncle over perineal region 3 weeks prior to this episode which was drained at a local hospital.

On physical examination, his BP was $100 / 60 \mathrm{~mm}$ $\mathrm{Hg}$, pulse was $120 \mathrm{bpm}$ and body temperature of $39^{\circ} \mathrm{C}$. On local examination of the back, he had tenderness over upper thoracic region with no local palpable mass/swelling, or local rise in temperature. He had normal motor power in both upper and lower extremities, and no sensory deficit. Bowel and bladder habits were normal. He exhibited exaggerated knee and ankle reflexes with well sustained clonus in his both lower limbs. Signs of meningeal irritations were absent. His cranial nerve examinations were normal.

Hematological investigation revealed a WBC count of 16,000 cells per microliter (Neutrophils $85 \%$ ), ESR - $124 \mathrm{~mm} / \mathrm{h}$ and CRP - $90 \mathrm{mg} / \mathrm{L}$. MRI dorsal spine was done. 
With the preliminary diagnosis of epidural abscess with paraspinal extension we planned for decompression. Epidural abscess was drained after wide laminectomy from D3-D6 level. Granulation tissue was noted in the epidural space compressing the cord at D3 to D6 level which was decompressed posteriorly. Posterior instrumentation was done at D3 and D6 level. Pus along with granulation tissue sample was sent for culture/sensitivity and biopsy. Methicillin resistant Staphylococcus aureus (MRSA) was isolated which was sensitive to Vancomycin. Patient was given 10 days of IV Vancomycin and discharged on oral Flucloxacillin for another 2 weeks. Post-operative period was uneventful with healthy and dry wound. Suture removal was done on the $14^{\text {th }}$ post-operative day.

Patient again presented to our clinic on the $28^{\text {th }}$ post-operative day with complaints of upper back pain and high grade fever. Two pustules were present $\sim 1 \mathrm{~cm}$ in diameter on proximal and distal end of the surgical scar. Patient was admitted and intravenous Vancomycin was restarted. Repeat MRI was done. Blood parameters showed - WBC count of 15,500 cells per microliter (Neutrophils $86 \%$ ), ESR - $96 \mathrm{~mm} / \mathrm{h}$ and CRP - $90 \mathrm{mg} / \mathrm{L}$.

IV Vancomycin was continued for 2 weeks and he was discharged on oral Erythromicin which was sensitive to the organism (MRSA) isolated from pustule. His fever subsided and he had no back pain after few days of antibiotics administration.

\section{Discussion}

The current annual incidence of SEA is estimated to be $2.5-3$ per 10,000 hospital admissions ${ }^{3}$.

Its incidence is increasing due to ageing population, increasing number of spinal surgeries, minimally invasive procedures and increased access to better diagnostic imaging modalities ${ }^{3}$. At the beginning of the twentieth century, mortality for SEA approached $100 \%$, whereas with the advent of effective antibiotics and advanced technology the mortality has been reduced to $15-20 \%{ }^{1}$. Death occurs due to overwhelming sepsis and is more common in patients with multiple comorbidities $^{1}$. About one-third of survivors may have a poor neurological outcome which may be due to diagnostic delays. A patient's ultimate neurological outcome correlates strongly with the severity and duration of neurological deficits prior to surgery 4 . The majority of SEA occur posteriorly $(80 \%)$, and are usually related to a distant infectious focus like pharyngitis, skin and soft tissue infections ${ }^{2}$. Anterior infections are generally related to osteomyelitis or diskitis. SEA is more common in thoracic and lumbar spine whereas only $20 \%$ of cases are seen in the cervical spine ${ }^{2}$.

Gadolinium-enhanced $\mathrm{MRI}$ is recommended as the most useful diagnostic test that should be performed promptly in any patient with clinical

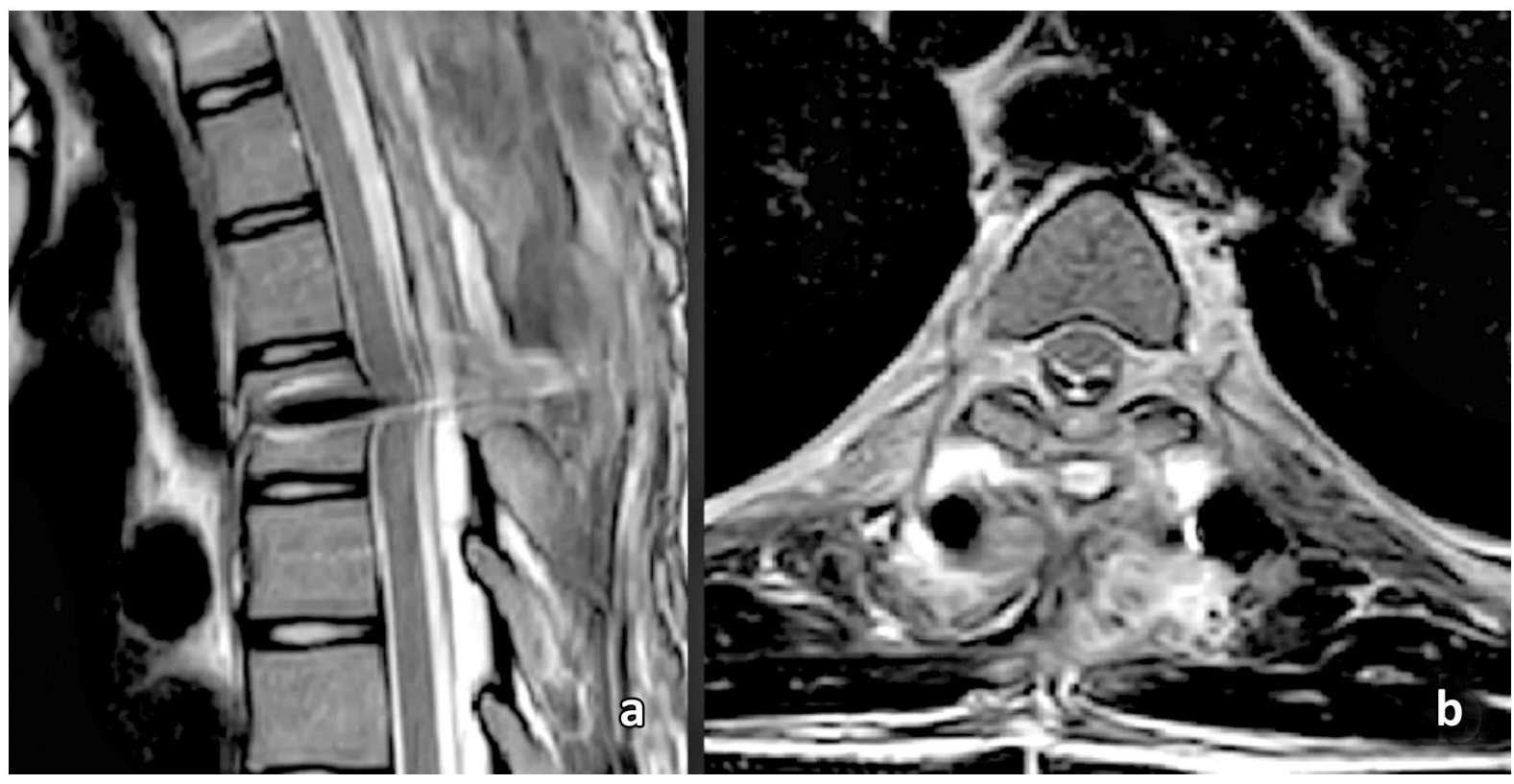

Figure 2: a, b -Status post laminectomy and fixation. Post-operative changes at D2 to D5 levels. No evidence of residual epidural abscess. Heterogeneous increased signal intensity in paravertebral regions with minimal collection at C7 to D6 level - post-operative changes. Marked departure from images in Fig. 1 at the same level. 
suspicion of SEA since early surgical drainage and appropriate antibiotics contribute to a favorable outcome $^{5}$. As any delay in treatment may contribute to irreversible neurological damage or death, once the diagnosis is suspected, prompt treatment is recommended. A blood culture should be obtained to confirm the diagnosis ${ }^{6}$. About $60 \%$ of the patients have bacteremia. Early surgical intervention in patients without neurological deficits have demonstrated favorable outcome ${ }^{8}$. In addition, even in patients with neurological deficits, if early decompression is performed within 24 hours of onset of neurological deficit, favorable outcome can be expected ${ }^{9}$. Complete loss of muscle activity (paralysis) lasting longer than 24-36 hours is unlikely to improve.

In the literature, the total duration of antibiotic therapy varies between 4 and 16 weeks, depending on co-morbidities, type of treatment (medical vs. surgical), isolated microorganism, bactericidal effect of the available agent and concomitant spinal infection ${ }^{3,4,7,8}$. Generally, resolution of the abscess is achieved after 4-6 weeks of treatment. In the case of concomitant vertebral osteomyelitis, treatment is prolonged as long as 8-12 weeks ${ }^{9}$. It is important to note that IV antibiotic therapy for the vertebral osteomyelitis for a duration of less than 4 weeks might lead to treatment failure ${ }^{11}$. Recommended optimal duration of parenteral antibiotics in patients without vertebral osteomyelitis is usually 2 to 4 weeks. If bony involvement is also observed, then the duration of the therapy should be extended to 6 to 8 weeks. During the treatment course, persistence of an elevated CRP is a prognostic factor $^{12}$

\section{Conclusion}

Best treatment of spinal epidural abscess even when there is no neurological deficit is drainage of the abscess. Posterior approach and laminectectomy is the standard way to achieve optimum drainage. The need of fixation depends on the amount of laminectomy, presence of vertebral body osteomyelitis, and anterior column destruction.
Administration of antibiotics should be continued for at least 6 weeks. Shorter duration of antibiotics even after surgical drainage could result in relapse of the disease as experienced in our case.

\section{References}

1. Reihsaus $E$, Waldbaur $H$, Seeling $W$. Spinal epidural abscess: A meta-analysis of 915 patients. Neurosurg Rev. 2000;23(4):175-204.

2. Baker AS, Ojemann RG, Swartz MN, Richardson EP Jr. Spinal epidural abscess. N Engl J Med 1975; 293(10): 463-8.

3. Sampath Rigamonti D. Spinal epidural abscess: A review of epidemiology, diagnosis, and treatment. J Spinal Disord. 1999;12(2):89-93.

4. Sendi P, Bregenzer T, Zimmerli W. Spinal epidural abscess in clinical practice. QJM. 2008;101(1):1-12.

5. Teman AJ. Spinal epidural abscess. Early detection with gadolinium magnetic resonance imaging. Arch Neurol 1992;49(7):743-6

6. Kabbara A, Rosenberg SK, Untal C. Methicillinresistant Staphylococcus aureus epidural abscess after transforaminal epidural steroid injection. Pain Physician. 2004;7(2):269-72.

7. Darouiche RO, Hamill RJ, Greenberg SB, Weathers SW, Musher DM. Bacterial spinal epidural abscess. Review of 43 cases and literature survey. Medicine 1992;71(6):369-85.

8. Sampath P, Rigamonti D. Spinal epidural abscess: a review of epidemiology, diagnosis, and treatment. J Spinal Disord. 1999;12(2):89-93.

9. Bluman EM, Palumbo MA, Lucas PR. Spinal epidural abscess in adults. J Am Acad Orthop Surg. 2004;12(3):155-63.

10. Soehle M, Wallenfang T. Spinal epidural abscesses: Clinical manifestations, prognostic factors, and outcomes. Neurosurgery. 2002;51(1):86-7.

11. Shuford JA, Steckelberg JM. Role of oral antimicrobial therapy in the management of osteomyelitis. Curr Opin Infect Dis. 2003;16(6):515-9.

12. Pereira CE, Lynch JC. Spinal epidural abscess: An analysis of 24 cases. Surg Neurol. 2005;63(suppl 1) S26-9. 